\title{
Insights into the role of hydration in protein structure and stability obtained through hydrostatic pressure studies
}

C.A. Royer
Centre de Biochimie Structurale, Montpellier Cedex, France

\author{
Correspondence \\ C.A. Royer \\ Centre de Biochimie Structurale \\ 29 rue de Navacelles \\ 34090 Montpellier Cedex \\ France \\ E-mail: royer@cbs.cnrs.fr \\ Presented at the 3rd International \\ Conference on High Pressure \\ Bioscience and Biotechnology, \\ Rio de Janeiro, RJ, Brazil, \\ September 27-30, 2004. \\ $\ldots \ldots \ldots \ldots$
}

Received December 17, 2004 Accepted May 12, 2005 ......................

\begin{abstract}
A thorough understanding of protein structure and stability requires that we elucidate the molecular basis for the effects of both temperature and pressure on protein conformational transitions. While temperature effects are relatively well understood and the change in heat capacity upon unfolding has been reasonably well parameterized, the state of understanding of pressure effects is much less advanced. Ultimately, a quantitative parameterization of the volume changes (at the basis of pressure effects) accompanying protein conformational transitions will be required. The present report introduces a qualitative hypothesis based on available model compound data for the molecular basis of volume change upon protein unfolding and its dependence on temperature.
\end{abstract}

Key words

- Pressure perturbation

calorimetry

- Folding

- Thermal expansivity

- Volume change

\section{Introduction}

In discussions of the use of pressure to study protein stability, it has often been suggested that pressure is a better variable than temperature or chemical denaturants for a variety of reasons. The implication of such statements is that one should preferentially carry out pressure perturbation studies in order to obtain some useful information. Clearly a large body of knowledge of protein structure and stability has been amassed using alternative approaches. Rather, a fundamental understanding of the behavior and properties of matter requires a fundamental characterization and comprehension of the response of the matter under consideration to the two fundamental thermodynamic variables, temperature and pressure. No further justification of the use of pressure to investi- gate the physicochemical properties of proteins is necessary. A detailed understanding of what pressure does to proteins and the molecular basis for these effects will provide invaluable insight into the factors (particularly hydration) that control protein structure and stability, an insight that is complementary to that obtained using temperature.

\section{What does pressure do to proteins?}

While in recent years a number of studies from many of the contributors to this conference, as well as others, have provided a significant amount of reliable data describing the physical effect of pressure on protein structure, the molecular basis for these changes has remained elusive. Prior to any discussion of the molecular basis for pressure effects it is important to present clearly 
what pressure does to proteins. Too often in the past, I and my colleagues in the field have exhibited the tendency to generalize the effects we have observed for a particular protein, under particular conditions, to proteins in general, under all conditions. Therefore, first of all it is important to stress that the only general comment one can make about the effect of pressure on protein structure is that it tends to favor protein conformations that occupy smaller specific volumes. Which of the conformations in its energy landscape a protein will adopt under particular conditions of temperature, pressure and solution composition depend upon the energy landscape of the protein, and implicate the relative stabilities (enthalpy and entropy), heat capacities, specific volumes, compressibilities, and expansivities of all of the possible configurations of the chain under those particular conditions. Thus, some proteins such as bovine pancreatic trypsin inhibitor, for example (1-8), undergo no major conformational changes upon application of pressures that are attainable in liquid type cells. The only observed changes in any spectroscopic property related to the structure of the protein indicate that pressure simply results in the compression of the native state of this protein. For other protein systems, the relative free energies and differences in specific volume between the native, folded state and conformational states that lie near this state and that have been referred to as low-lying excited states by Akasaka and $\mathrm{Li}$ (9) are such that the application of relatively modest pressures leads to the non-negligible population of these states. When the energy landscape of proteins is such that the population of folding intermediates (differentiated from the low-lying excited states by a significantly higher degree of disorder) is not so highly unfavorable, pressure will often favor such states because their specific volumes are intermediate between that of the folded state and the unfolded state, and their relative stability ren- ders them accessible by pressure perturbation. This is clearly the case for apo-myoglobin (9-15), for which moderate pressure results in the population of a species with structural characteristics quite similar to those of the intermediates observed by chemical perturbations. Finally, a relatively large percentage of the proteins that have been studied using pressure perturbations can be unfolded completely in a pressure range accessible in liquid cells ( $<10 \mathrm{kbar})(16-26)$. Although it is important to note that the meaning of the phrase completely unfolded is subject to debate, can change with temperature and solution conditions and depends upon the protein under study, and this regardless of the perturbation used. In any case, one must take care in stating what it is that pressure does to proteins, given that this depends (first of all) upon the protein, and then upon the conditions under which the pressure has been applied. One very important parameter that is often ignored in discussions of pressure effects is the concentration of the protein. Often dictated by the technique used to observe the effects of pressure, changes in this parameter can lead to very different observations, since aggregation phenomena are favored at high concentrations. Thus, pressure-induced aggregation is often observed in high-pressure Fourier transform infrared studies in which high concentrations of protein are required (27-32). In one case at high-Fourier transform infrared concentration, pressure led to the population of a partially unfolded dimer, whereas for the same system at the lower concentrations used in fluorescence, pressure led to total dissociation and unfolding (26).

\section{Why does pressure do what it does?}

In the following discussion of the underlying contributions to volume changes for protein conformational transitions, we will refer only to the transitions between folded and unfolded structures, since these transi- 
tions involve the entire protein structure and are thus the largest in magnitude. Despite the elusive nature of the underlying causes of the effects of pressure on protein structure, we understand in general what factors make a contribution. First, compression is relatively straightforward. All conformational states of proteins will undergo compression upon application of pressure. Such changes can occur up to the point at which it is energetically more favorable to undergo a transition to a different state (unfolded, intermediate, aggregate). This situation is analogous to that of the effect of temperature, in which heating leads to expansion of the native state until, at some temperature, the protein unfolds. Hydration changes that accompany protein conformational transitions are largely responsible for pressureinduced protein structural transitions. Differences in specific volume between protein conformational states constitute the basis for the effects of pressure on the equilibrium between these states. Unarguably, proteins are highly densely packed in their folded states. Nonetheless, their interiors present void volumes that exclude solvent and protein moieties due to their size and distribution in the structure. Thus, the opening up of protein structure allowing a solvent to occupy an internal volume previously excluded from interaction with it constitutes a clear contributor to changes in the degree of hydration upon changes in conformation. Secondly, unfolded proteins present overall more surface area to the solvent, and thus their degree of solvation is larger than for folded proteins. In addition, folded proteins bury a higher percentage of non-polar as opposed to polar residues in their native structures, and hence, the nature of the hydration of the protein surface is different in the folded state compared to more open, disordered states. Thus, both the degree and type of hydration will contribute to the differences in specific volume between folded and unfolded states. Whether these differences in hydration re- sult in overall decreases or increases in specific volume accompanying the transition will depend upon the type of hydration and the temperature at which the unfolding transition occurs.

We know that changes in solvation between protein conformational states constitute the fundamental basis of the effects of pressure on protein structure, but we are a long way from a quantitative parameterization of these volume changes (33-39). One problem in quantifying the underlying contributions to the volume change is centered upon how to evaluate the contribution of internal cavities to the value of the volume change upon unfolding. Some success has come from experimental attempts at making a quantitative measurement of this effect $(22,40)$. The magnitude and even the sign of the volume change associated with hydrophobic hydration are still under debate $(36,38)$. And while the solvation of polar moieties is thought to contribute to an overall decrease in volume, the magnitude of this effect is difficult to predict.

Experimentally it has been observed fairly consistently that the volume change upon unfolding, which is typically negative and relatively large in magnitude $(0.5-1 \%$ of the total protein volume) at low temperature, decreases in absolute value with increasing temperature (41-43). This change in the volume change of unfolding as a function of temperature corresponds to the difference in thermal expansivity between the folded and unfolded states, and indicates that the expansivity of the unfolded state is larger than that of the folded state, experimentally by about $1 \times 10^{-3} \mathrm{~K}^{-1}$. Using densitometry, we have measured the volume of staphylococcal nuclease as a function of both temperature and pressure, allowing the determination of both the expansivity and compressibility of the folded and unfolded states under conditions of temperature and pressure where each is stable (44). It is clear from our results that the volume of the folded state 
Figure 1. Expansivities of amino acids as a function of temperature determined by pressure perturbation calorimetry (PPC). The values of $\alpha$ were estimated from the data presented by Brandts and co-workers (45) and plotted on the same graph for comparison. $\mathrm{T}=$ temperature. does not increase linearly with temperature, i.e., that the expansivity of the folded state is temperature dependent.

Brandts and co-workers (45) introduced the pressure perturbation calorimeter (PPC), which permits a direct measurement of the change in heat capacity as a function of pressure. Since this quantity corresponds to the opposite of the thermal expansivity, $\alpha$, at the temperature studied multiplied by the temperature and the volume at that temperature, measurement of the heat released or taken up upon a small pressure perturbation yields the value of the expansivity. Measurements are carried out over a broad temperature range, and integration of the expansivity over the temperatures of the unfolding transition yields the volume change of the transition (at the transition temperature).

As demonstrated by Brandts et al. (45), at $3^{\circ} \mathrm{C}$, water exhibits a negative expansivity, i.e., the heat capacity of water increases as the pressure is raised. A negative value of $\alpha$ can be understood at the molecular level as follows: at this low temperature, water is highly structured, much like ice. As it is heated, the structure breaks down and the density of the water actually increases. This effect is large enough to overcome the tem-

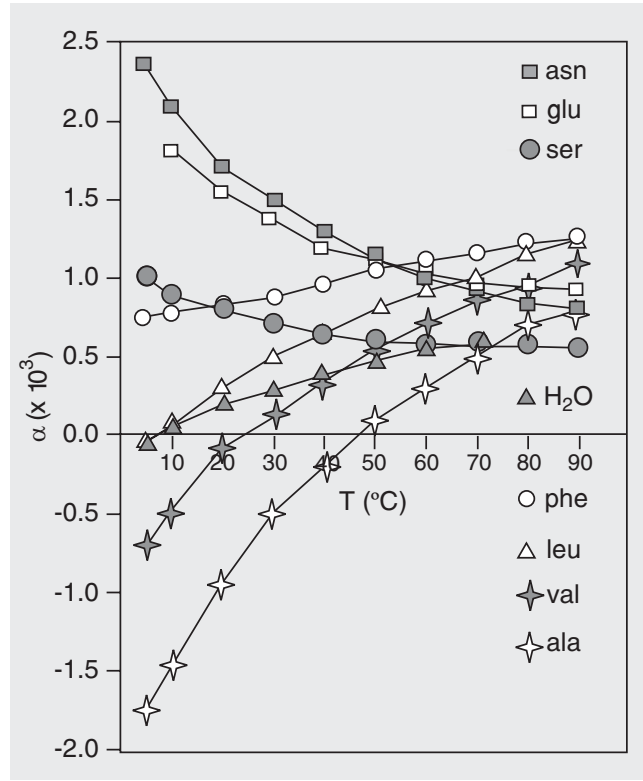

perature-induced increases in bond length. Likewise, the increase in heat capacity with pressure at this temperature can be understood in the same light. As pressure is increased, the denser more disordered structure of water is favored, and this structure has a higher heat capacity than does the ordered structure, which exhibits fewer degrees of freedom. Above $4^{\circ} \mathrm{C}$, the expansivity of water becomes positive because the low density structure is melting out and the effects of thermal motion come to dominate. Addition of solutes (such as proteins) to water will alter the expansivity in a manner that depends upon whether the solutes favor the denser or the more structured form of their hydrating water.

In discussing expansivity of proteins one must distinguish between the expansivity of the protein chain itself, and the expansivity due to hydration. The intrinsic expansivity of the folded chain has been shown to be smaller than the expansivity of bulk solvent (46-48) and is limited by the interactions of the folded structure. The intrinsic expansivity of the unfolded chain is difficult to predict since this will depend upon the residual structure in the unfolded state. It is assumed here that the major contribution to the expansivities of both the folded and the unfolded states arises from hydration effects. Brandts and co-workers (45) have measured the expansivities of hydrophobic, polar and aromatic amino acids (Figure 1). Like most other aspects of protein thermodynamics (and as one should have expected), it would appear that actual values of the thermal expansivities of proteins result from the combination of relatively large and opposite effects $(45,49,50)$.

The expansivity of folded proteins appears to be dominated by the contributions of polar amino acids since the values are positive (near $1 \times 10^{-3} \mathrm{~K}^{-1}$ at around $4^{\circ} \mathrm{C}$ ) and decreases in a fashion similar to the curve of serine in Figure 1 before undergoing temperature-induced unfolding. The expansivity 
of unfolded proteins has only been measured at the very high temperatures at which unfolded states are populated. Since unfolded proteins expose a larger fraction of hydrophobic surface area to the solvent than do folded proteins, one might imagine that the expansivity of unfolded proteins might be lower than that of folded proteins at lower temperature and higher than that of folded proteins at high temperature. Thus, the overall expansivity of the unfolded state will have a positive contribution to the temperature dependence of $\alpha$ from the exposed hydrophobic residues. This will tend to attenuate the negative contribution to the temperature dependence of $\alpha$ from the polar residues. Thus, overall, the temperature dependence of $\alpha$ (Figure 2) for the unfolded state may be smaller than that for the folded state. Of course, if this is true, then $\Delta \alpha$ is not constant with temperature. Such an idea is borne out by the measure of the expansivity of a solvated hydrophobic elastin-like peptide GVG(VPGVG) (51,52), which is largely unstructured below $40^{\circ} \mathrm{C}$ and undergoes a transition to a ß-type structure as the temperature is increased. Between 0 and $20^{\circ} \mathrm{C}$, the decrease in $\alpha$ for this peptide is very small (1.05 to $0.8510^{-3} \mathrm{~K}^{-1}$ ).

Given these considerations, we can begin to raise some hypotheses about the contributions of hydrophobic hydration, polar hydration and cavities to the value of the volume change as a function of temperature. At low temperature, the hydration of hydrophobic residues exposed upon unfolding should lead to an increase in volume because the density of the water around the polar residues is smaller than the bulk. But, at the same time, exposure of polar residues and their subsequent hydration, as well as the elimination of cavities should lead to a decrease in volume. As the temperature increases, because the expansivity of the folded state has a stronger negative temperature dependence than does the unfolded state, the volume of the folded state increases signifi-

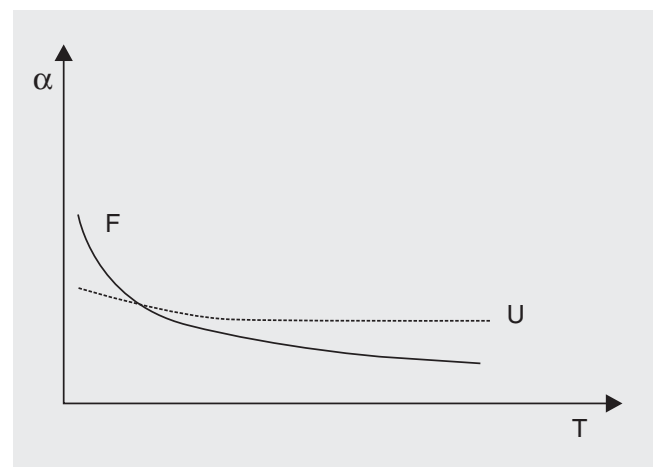

Figure 2. Hypothetical plots of the temperature $(\mathrm{T})$ dependence of the expansivities of the folded (F) and unfolded (U) states based on the behavior of model compounds.

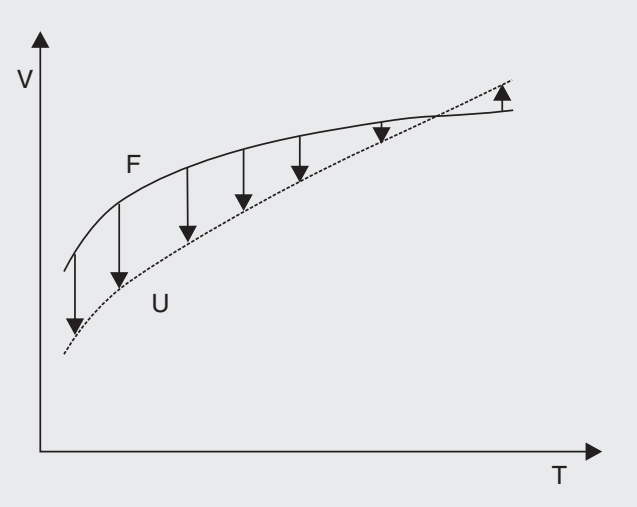

Figure 3. Hypothetical temperature dependence of the volumes of the folded $(F)$ and unfolded $(U)$ states based on the hypothetical temperature $(\mathrm{T})$ dependence of $\alpha$ in Figure 2. ( $\Delta \mathrm{Vu}$ is given by the arrows at several temperatures). cantly at low temperature, but much less so as temperature is raised. In the meantime, the volume of the unfolded state, while much lower than that of the folded state at low temperature, should increase more steadily as the temperature is raised, thus diminishing the differences between the two and eventually leading to a change in sign of $\Delta \mathrm{Vu}$ (Figure 3). Thus, generally the dependence of $\Delta \mathrm{Vu}$ on temperature is due to the increasing role of hydrophobic hydration and the decreasing role of polar hydration as temperature is raised.

Presented here is a qualitative proposal for the temperature dependence of the pressure effects on proteins. Confirming this hypothesis will require new densitometric and pressure perturbation calorimetry data for more model compounds, small peptides of varying composition and finally very small proteins. This would allow us to begin to quantitatively parameterize the contributions 
of hydration to the specific volumes of folded and unfolded states of proteins as a function of temperature. The contribution of cavities to the overall volume change can be estimated based on estimation of the intrinsic volume of the cavities from X-ray crystallo- graphic data. In addition it will be important to take into consideration the type of residues exposed upon cavity elimination and the temperature dependence of their hydration contributions to the specific volume.

\section{References}

1. Akasaka K, Li H, Yamada $\mathrm{H}$ et al. (1999). Pressure response of protein backbone structure. Pressure-induced amide $15 \mathrm{~N}$ chemical shifts in BPTI. Protein Science, 8: 1946-1953.

2. Goossens K, Smeller L, Frank J et al. (1996). Pressure-tuning the conformation of bovine pancreatic trypsin inhibitor studied by Fourier-transform infrared spectroscopy. European Journal of Biochemistry, 236: 254-262.

3. Kitchen DB, Reed LH \& Levy RM (1992). Molecular dynamics simulation of solvated protein at high pressure. Biochemistry, 31: 1008310093.

4. Li H, Yamada H \& Akasaka K (1998). Effect of pressure on individual hydrogen bonds in proteins. Basic pancreatic trypsin inhibitor. Biochemistry, 37: 1167-1173.

5. Li H, Yamada H \& Akasaka K (1999). Effect of pressure on the tertiary structure and dynamics of folded basic pancreatic trypsin inhibitor. Biophysical Journal, 77: 2801-2812.

6. Sareth S, Li H, Yamada $\mathrm{H}$ et al. (2000). Rapid internal dynamics of $\mathrm{BPTI}$ is insensitive to pressure. (15) N spin relaxation at 2 kbar. FEBS Letters, 470: 11-14.

7. Takeda N, Nakano K, Kato M et al. (1998). Pressure-induced structural rearrangements of bovine pancreatic trypsin inhibitor studied by FTIR spectroscopy. Biospectroscopy, 4: 209-216.

8. Williamson MP, Akasaka K \& Refaee M (2003). The solution structure of bovine pancreatic trypsin inhibitor at high pressure. Protein Science, 12: 1971-1979.

9. Akasaka K \& Li H (2001). Low-lying excited states of proteins revealed from nonlinear pressure shifts in $1 \mathrm{H}$ and $15 \mathrm{~N}$ NMR. Biochemistry, 40: 8665-8671.

10. Bismuto E, Sirangelo I, Irace G et al. (1996). Pressure-induced perturbation of apomyoglobin structure: fluorescence studies on native and acidic compact forms. Biochemistry, 35: 1173-1178.

11. Bismuto E, Irace G, Sirangelo I et al. (1996). Pressure-induced perturbation of ANS-apomyoglobin complex: frequency domain fluorescence studies on native and acidic compact states. Protein Science, 5: 121-126.

12. Bondos SE, Sligar S \& Jonas J (2000). High-pressure denaturation of apomyoglobin. Biochimica et Biophysica Acta, 1480: 353-364.

13. Kitahara R, Yamada $H$, Akasaka K et al. (2002). High pressure NMR reveals that apomyoglobin is an equilibrium mixture from the native to the unfolded. Journal of Molecular Biology, 320: 311-319.

14. Sire O, Alpert B \& Royer CA (1996). Probing $\mathrm{pH}$ and pressure effects on the apomyoglobin heme pocket with the 2'-(N,N-dimethylamino)-6-naphthoyl-4-trans-cyclohexanoic acid fluorophore. Biophysical Journal, 70: 2903-2914.

15. Vidugiris GJ \& Royer CA (1998). Determination of the volume changes for pressure-induced transitions of apomyoglobin between the native, molten globule, and unfolded states. Biophysical Journal,
75: 463-470.

16. Ruan Q, Ruan K, Balny C et al. (2001). Protein folding pathways of adenylate kinase from E. coli: hydrostatic pressure and stoppedflow studies. Biochemistry, 40: 14706-14714.

17. Ruan K, Xu C, Yu Y et al. (2001). Pressure-exploration of the 33$\mathrm{kDa}$ protein from the spinach photosystem II particle. European Journal of Biochemistry, 268: 2742-2750.

18. Royer CA, Hinck AP, Loh SN et al. (1993). Effects of amino acid substitutions on the pressure denaturation of staphylococcal nuclease as monitored by fluorescence and nuclear magnetic resonance spectroscopy. Biochemistry, 32: 5222-5232.

19. Panick G \& Winter R (2000). Pressure-induced unfolding/refolding of ribonuclease A: static and kinetic Fourier transform infrared spectroscopy study. Biochemistry, 39: 1862-1869.

20. Oliveira AC, Gaspar LP, Da Poian AT et al. (1994). Arc repressor will not denature under pressure in the absence of water. Journal of Molecular Biology, 240: 184-187.

21. Mei G, Di VA, Campeggi FM et al. (1999). The effect of pressure and guanidine hydrochloride on azurins mutated in the hydrophobic core. European Journal of Biochemistry, 265: 619-626.

22. Lassalle MW, Yamada H, Morii $\mathrm{H}$ et al. (2001). Filling a cavity dramatically increases pressure stability of the c-Myb R2 subdomain Proteins, 45: 96-101.

23. Kitahara R, Royer C, Yamada $\mathrm{H}$ et al. (2002). Equilibrium and pressure-jump relaxation studies of the conformational transitions of P13MTCP1. Journal of Molecular Biology, 320: 609-628.

24. Jacob MH, Saudan C, Holtermann G et al. (2002). Water contributes actively to the rapid crossing of a protein unfolding barrier. Journal of Molecular Biology, 318: 837-845.

25. Herberhold H, Marchal S, Lange R et al. (2003). Characterization of the pressure-induced intermediate and unfolded state of red-shifted green fluorescent protein - a static and kinetic FTIR, UV/VIS and fluorescence spectroscopy study. Journal of Molecular Biology, 330: 1153-1164.

26. Desai G, Panick G, Zein M et al. (1999). Pressure-jump studies of the folding/unfolding of trp repressor. Journal of Molecular Biology, 288: 461-475

27. Goossens K, Haelewyn J, Meersman F et al. (2003). Pressure- and temperature-induced unfolding and aggregation of recombinant human interferon-gamma: a Fourier transform infrared spectroscopy study. Biochemical Journal, 370: 529-535.

28. Meersman F, Smeller L \& Heremans K (2002). Comparative Fourier transform infrared spectroscopy study of cold-, pressure-, and heatinduced unfolding and aggregation of myoglobin. Biophysical Journal, 82: 2635-2644.

29. Meersman F \& Heremans K (2003). High pressure induces the formation of aggregation-prone states of proteins under reducing 
conditions. Biophysical Chemistry, 104: 297-304.

30. Ruan K, Lange R, Meersman F et al. (1999). Fluorescence and FTIR study of the pressure-induced denaturation of bovine pancreas trypsin. European Journal of Biochemistry, 265: 79-85.

31. Scheyhing $\mathrm{CH}$, Meersman F, Ehrmann MA et al. (2002). Temperature-pressure stability of green fluorescent protein: a Fourier transform infrared spectroscopy study. Biopolymers, 65: 244-253.

32. Smeller L, Rubens P \& Heremans K (1999). Pressure effect on the temperature-induced unfolding and tendency to aggregate of myoglobin. Biochemistry, 38: 3816-3820.

33. Balny C, Masson P \& Heremans K (2002). High pressure effects on biological macromolecules: from structural changes to alteration of cellular processes. Biochimica et Biophysica Acta, 1595: 3-10.

34. Demchenko AP (2001). Concepts and misconcepts in the analysis of simple kinetics of protein folding. Current Protein and Peptide Science, 2: 73-98.

35. Heremans K (1982). High pressure effects on proteins and other biomolecules. Annual Review of Biophysics and Bioengineering, 11: 1-21.

36. Mozhaev VV, Heremans K, Frank J et al. (1996). High pressure effects on protein structure and function. Proteins, 24: 81-91.

37. Ooi T (1994). Thermodynamics of protein folding: effects of hydration and electrostatic interactions. Advances in Biophysics, 30: 105154.

38. Royer CA (2002). Revisiting volume changes in pressure-induced protein unfolding. Biochimica et Biophysica Acta, 1595: 201-209.

39. Silva JL \& Weber G (1993). Pressure stability of proteins. Annual Review of Physical Chemistry, 44: 89-113.

40. Frye KJ \& Royer CA (1998). Probing the contribution of internal cavities to the volume change of protein unfolding under pressure. Protein Science, 7: 2217-2222.

41. Brandts JF, Oliveira RJ \& Westort C (1970). Thermodynamics of protein denaturation. Effect of pressure on the denaturation of ribonuclease A. Biochemistry, 9: 1038-1047.

42. Panick G, Vidugiris GJ, Malessa R et al. (1999). Exploring the temperature-pressure phase diagram of staphylococcal nuclease.
Biochemistry, 38: 4157-4164.

43. Zipp A \& Kauzmann W (1973). Pressure denaturation of metmyoglobin. Biochemistry, 12: 4217-4228.

44. Seemann H, Winter R \& Royer CA (2001). Volume, expansivity and isothermal compressibility changes associated with temperature and pressure unfolding of staphylococcal nuclease. Journal of Molecular Biology, 307: 1091-1102.

45. Lin LN, Brandts JF, Brandts JM et al. (2002). Determination of the volumetric properties of proteins and other solutes using pressure perturbation calorimetry. Analytical Biochemistry, 302: 144-160.

46. Rholam M, Scarlata S \& Weber G (2004). Frictional resistance to local rotation of fluorophores in proteins. Biochemistry, 23: 67936796.

47. Scarlata S, Rholam M \& Weber G (2004). Frictional resistance to local rotations of aromatic fluorophores in some small peptides. Biochemistry, 23: 6789-6792.

48. Weber G, Scarlata S \& Rholam M (2004). Thermal coefficient of the frictional resistance to rotation in simple fluorophores determined by fluorescence polarization. Biochemistry, 23: 6785-6788.

49. Ravindra R \& Winter R (2004). Pressure perturbation calorimetry: a new technique provides surprising results on the effects of cosolvents on protein solvation and unfolding behaviour. Chemphyschem, 5: 566-571.

50. Ravindra R, Royer C \& Winter R (2004). Pressure perturbation calorimetric studies on the solvation properties and the thermal unfolding of staphylococcal nuclease. Physical Chemistry Chemical Physics, 6: 1952-1961.

51. Nicolini C, Ravindra R, Ludolph B et al. (2004). Characterization of the temperature- and pressure-induced inverse and reentrant transition of the minimum elastin-like polypeptide GVG(VPGVG) by DSC, PPC, CD, and FT-IR spectroscopy. Biophysical Journal, 86: 13851392.

52. Schreiner E, Nicolini C, Ludolph B et al. (2004). Folding and unfolding of an elastin-like oligopeptide: "inverse temperature transition," reentrance, and hydrogen-bond dynamics. Physical Review Letters, 92: 148101 